\title{
Respiratory insufficiency due to high anterior cervical cord infarction
}

\author{
R S Howard, J Thorpe, R Barker, T Revesz, N Hirsch, D Miller, A J Williams
}

\begin{abstract}
Objects and methods-Respiratory dysfunction may occur as a result of lesions in the upper cervical spinal cord disturbing the descending pathways subserving automatic and volitional ventilatory control. Four patients are described who presented with acute respiratory insufficiency caused by infarction of the anterior portion of the upper cervical cord due to presumed anterior spinal artery occlusion.

Results-Two patients presented after respiratory arrests; they were ventilated and there was no automatic or volitional respiratory effort. Both had signs of an extensive anterior spinal cord lesion at the C2 level and this was confirmed by MRI. One patient presented with a $\mathrm{C} 4$ infarction and required ventilation for three months. Ventilatory recovery was characterised by the development of an automatic respiratory pattern. The fourth patient required ventilation for two months after infarction at the C3 level. On attempted weaning he had prolonged periods of hypoventilation and apnoea during inattention and sleep indicating impairment of automatic respiratory control.
\end{abstract}

Conclusion-Infarction of the spinal cord at high cervical levels may be due to fibrocartilaginous embolism and involvement of the descending respiratory pathways may occur. Extensive lesions at $\mathrm{C} 1 / 2$ cause complete interruption of descending respiratory control leading to apnoea. Partial lesions at $\mathrm{C} 3 / 4$ cause selective interruption of automatic or voluntary pathways and give rise to characteristic respiratory patterns. The prognosis depends on the level and extent of the lesion.

(F Neurol Neurosurg Psychiatry 1998;64:358-361)

Keywords: cervical cord infarction; respiratory insufficiency; anterior spinal artery occlusion

Traumatic or demyelinating lesions of the spinal cord, particularly at high cervical levels, may selectively affect respiratory control. ${ }^{12}$ Studies have shown that tetraplegic patients are prone to disordered breathing in sleep, but whereas this is usually due to obstructive sleep apnoea, ${ }^{3}$ hypoventilation may occur as a consequence of impaired intercostal and diaphragmatic function. ${ }^{4}$ Although the pathogenesis and clinical manifestations of spinal cord infarction are clear, ${ }^{56}$ the effects of acute vascular lesions of the high cervical cord are less well described. We present four patients with severe respiratory insufficiency associated with infarction of the high cervical cord presumed to be due to occlusion of the anterior spinal artery. The patients were admitted to the Batten/Harris Neurological Intensive Care Unit or the Lane Fox Respiratory Unit, St Thomas' Hospital over an eight year period. Two patients presented with global respiratory impairment and one each with impairment of automatic and volitional control.

\section{Patients}

PATIENT

A 30 year old man presented with a sharp pain at the back of his neck after "working out" at a gymnasium. He developed paraesthesiae affecting his left forearm and within one hour this spread to involve the right forearm. He went to sleep and awoke with severe neck pain and limb weakness such that he was unable to lift his arms. He became progressively weaker while awaiting the ambulance and was unable to move his legs on arrival at the accident and emergency department. Within 10 minutes he complained of being breathless and was unable to take a breath to command. He then became apnoeic and had a respiratory arrest and was intubated and ventilated. On examination there was no neck stiffness and the cranial nerves were normal. The limbs were flaccid and there was severe weakness throughout but movements against gravity were preserved in all muscle groups. The reflexes were initially just present but later became brisk with flexor plantar responses. There was a sensory level to pin prick and temperature appreciation at C4 but proprioception was preserved. T2 Weighted MRI showed a high signal lesion affecting the anterior spinal cord and extending from $\mathrm{C} 1$ to $\mathrm{C} 6$ (fig 1). He remained ventilated with no volitional or automatic respiratory efforts. Two days after admission he had a cardiopulmonary arrest from which he could not be resuscitated. Necropsy confirmed a massive pulmonary embolism. Histological examination of the cervical spinal cord showed the presence of an infarct in the supply territory of the anterior spinal artery. The infarct was well demarcated, and showed some reactive capillary proliferation and an early macrophage invasion around the edges of the lesion. The ischaemic lesion affected the lateral one third of the anterior grey horn and some depletion of the anterior horn motor neurons was present. At $\mathrm{C} 1$ the ventral portions of the funiculus gracilis and cuneatus just dorsal to the anterior white commissures were affected. The lateral third of the anterior grey horn was also 


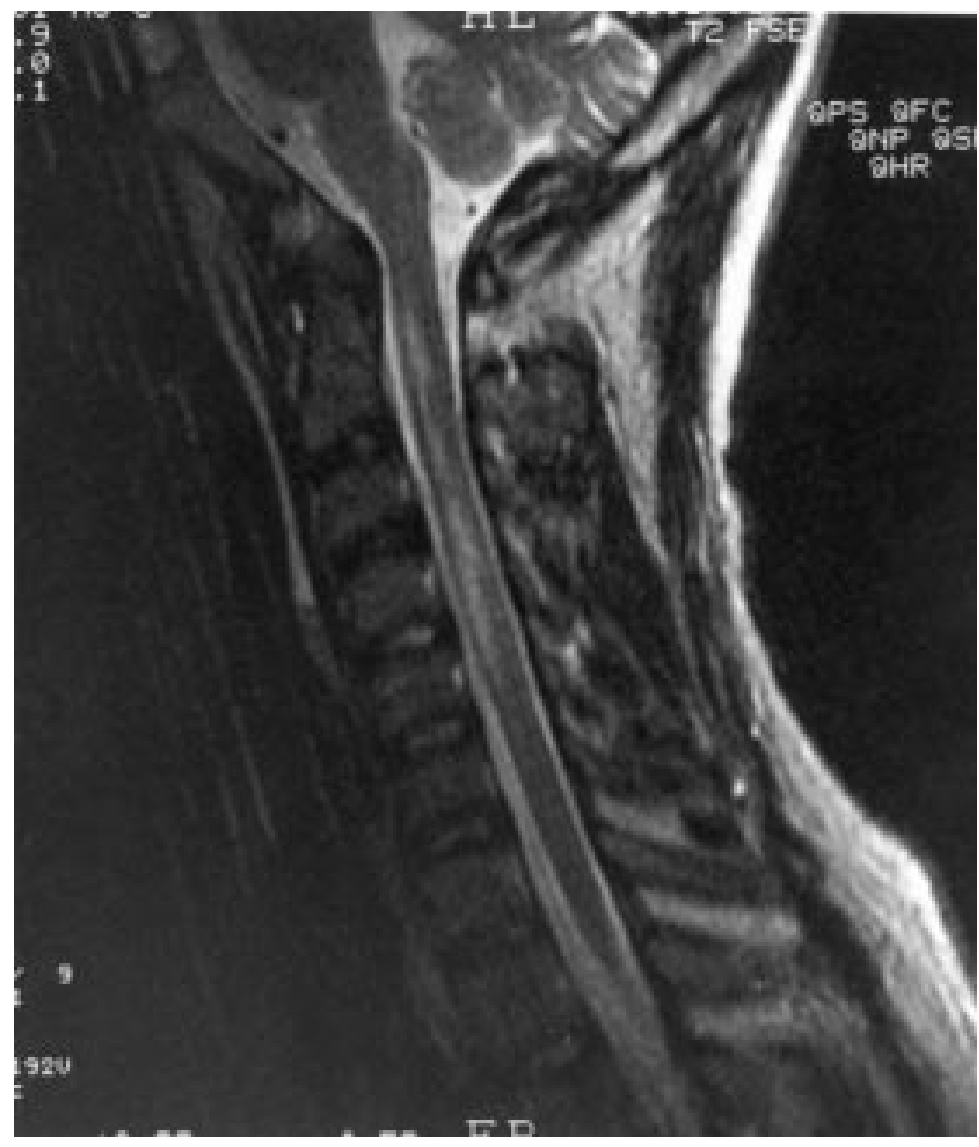

Figure 1 T2 Weighted MRI shows a high signal lesion affecting the anterior spinal cord and extending from $\mathrm{C} 1$ to $\mathrm{C} 6$. involved as were the anterior corticospinal tracts. The cord lesion, which was more severe on one side throughout, continued through C3 and $\mathrm{C} 4$ to become the most extensive at C6. Here the infarct occupied nearly the whole anatomical area of the lateral funiculus, on one side, with extensive involvement of the lateral corticospinal tract while only a small infarcted area was seen in this tract on the other side. The dorsal and ventral spinocerebellar and the lateral spinothalamic tracts were also affected on the side with the more severe damage. The lateral one third of the anterior grey horn was infarcted on both sides. The lesion of the cervical cord did not extend into the medulla (fig 2).

PATIENT 2

A 27 year old woman developed sudden onset of severe neck pain and ascending paraesthesiae affecting the legs and within six hours the arms. She was taken to the accident and emergency department where she complained of difficulty with breathing and then had a respiratory arrest. She was intubated and ventilated. On examination the cranial nerves were intact. The limbs were flaccid with no movement and areflexia throughout. There was diminished light touch and pin prick appreciation to $\mathrm{C} 2$ but proprioception was preserved; MRI showed an extensive, long, enhancing intrinsic cord lesion extending from $\mathrm{C} 1$ to $\mathrm{C} 7$ with no brainstem involvement.

One year after presentation she remained on continuous ventilatory support with no auto-

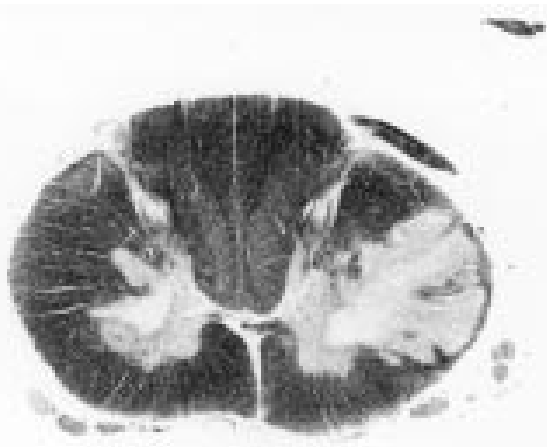

Figure 2 Histological examination of the cervical spinal cord at $C 6$ showing a well demarcated infarct affecting nearly the whole anatomical area of the lateral funiculus, on one side, with extensive involvement of the lateral corticospinal tract whereas only a small infarcted area is seen in this tract on the other side. The dorsal and ventral spinocerebellar and the lateral spinothalamic tracts are also affected on the side of the more severe damage. The lateral one third of the anterior grey horn is infarcted on both sides.

matic or volitional respiratory efforts. On examination the cranial nerves were normal. There were no voluntary movements but the limbs showed severe spasticity with hyperreflexia. There was a level to spinothalamic sensation at $\mathrm{C} 2$ but proprioception remained normal. Repeat MRI showed an atrophic spinal cord in the cervical region.

PATIENT 3

A 23 year old woman presented with a 24 hour history of sharp and severe neck pain. Three days later she developed progressive paraesthesiae and weakness affecting the right arm followed by the left arm and both legs. On examination there was a flaccid tetraplegia with no volitional movement, the reflexes were normal and there was a C4 sensory level to all modalities. One day later she had a respiratory arrest necessitating intubation and ventilation; MRI showed high signal at C3/4 in the central and anterior regions. After three months she was gradually weaned from ventilatory support. The respiratory pattern studied using inductance plethysmography (Respitrace, Ambulatory Monitoring Inc, NY, USA) showed an unvarying respiratory rate and tidal volume which could not be influenced in response to command or other volitional effort. The arterial blood gases during spontaneous ventilation were normal with a mean $\mathrm{PaCO}_{2}$ of 43 $\mathrm{mm} \mathrm{Hg}$. A hypercapnic response was not undertaken. The abnormal pattern resolved after two weeks. There was partial recovery of the tetraplegia and sensory loss but the patient remains wheelchair bound.

\section{PATIENT 4}

A 61 year old man presented with pain across the scapular region radiating into the right arm and partial weakness of the right leg with areflexia and an extensor plantar response on the right. The next morning he was unable to move the left arm and leg. He developed urinary retention and required catheterisation. 
The weakness progressed and examination showed flaccid tetraplegia with flexor spasms of the legs. The reflexes were preserved and plantar responses were flexor. There was an asymmetric disturbance of spinothalamic sensation up to $\mathrm{C} 2$ on the right and $\mathrm{C} 4$ on the left. Joint position and vibration sense were normal; MRI showed a high signal lesion at the $\mathrm{C} 3$ level confined to the anterior two thirds of the cord. $\mathrm{He}$ became increasingly breathless and required intubation and ventilation. $\mathrm{He}$ was weaned and extubated after two months. Chest wall and abdominal movements were orthodox, the forced vital capacity was 2.21 (predicted 3.11) but did not vary significantly between the erect and supine positions suggesting that function of the diaphragm was relatively well preserved. For the next three months ventilation was normal while awake but on sleeping he developed nocturnal hypoventilation and presumed central sleep apnoea with prolonged periods of desaturation on oximetry ( $>20$ minutes of $>5 \%$ below baseline saturation). His limb and respiratory function improved gradually and he was independently mobile one year after presentation.

\section{Discussion}

The most anterior part of the lateral columns of the first three cervical segments is critical for the maintenance of automatic respiratory activity in humans. Studies of patients undergoing high cervical cordotomies for relief of pain showed that section of the cord (high cervical cordotomy) in the region of the descending reticulospinal pathways selectively impairs spontaneous respiratory muscle activity on the ipsilateral side. ${ }^{7}$ Other reports have confirmed that high bilateral venterolateral cordotomy causes decreased tidal volume, vital capacity, and reduced ventilatory response to $\mathrm{CO}_{2}$ due to interruption of automatic respiration, culminating in nocturnal apnoea. ${ }^{8}{ }^{9}$ This pattern was seen in patient 4 who had MRI changes compatible with infarction of the anterior two thirds of the cord at the C3 level. Voluntary control of breathing is mediated by the cortical outflow to respiratory muscles in the dorsolateral corticospinal tracts. ${ }^{10}$ Selective interruption of these pathways in the spinal cord results in automatic breathing in much the same way as their interruption by bilateral corticospinal tract lesions in the basal pons or at the decussation. ${ }^{11} 12$ Patient 3 showed such automatic breathing with a strikingly regular and unvarying respiratory rate during which she was unable to take a deep breath, hold her breath, cough voluntarily, or initiate any kind of volitional respiratory movement. In this patient, as in those with multiple sclerosis, ${ }^{13}$ the severity of the loss of voluntary control of respiratory muscles paralleled that of limb muscles, suggesting that this system is served by descending tracts that lie together in the dorsolateral columns. More extensive bilateral lesions of the high cervical cord lead to an extensive involvement of all the lateral and anterior pathways and produce complete interruption of descending respiratory control (patients 1 and 2).The respiratory effects of vascular lesions of the spinal cord depend on the timing of onset and the extent of involvement of the phrenic nerve supply (C3-C5). Complete lesions usually lead to sudden respiratory arrest and death unless immediate resuscitation is available as in patients 1 and 2 . The anterior spinal artery runs in the ventral midline rostrally from the spinomedullary junction of the foramen magnum caudally to the tip of the spinal cord. At the upper cervical levels the most rostral supply to the cord comes from the vertebral arteries with a further supply from the posterior inferior cerebellar artery and the thyrocervical and costocervical branches of the subclavian artery at the cervical enlargement. An anastomotic network between the neck arteries contributes indirectly to the vascularisation of the lower cervical and upper thoracic cord. The anterior spinal artery supply is at its most marginal at the upper thoracic region (T2-4) and this thoracic watershed zone is vulnerable to hypotensive infarction. However, the pattern of cervical radicular arteries has led to the suggestion that a minor watershed zone also exists at $\mathrm{C} 4 .{ }^{6}$ Anterior spinal artery occlusion leads to a characteristic syndrome with the abrupt onset of weakness and, below the level of the lesion, flaccid paraplegia, areflexia, loss of spinothalamic perception of pain and temperature, and autonomic deficits, comprising sphincter flaccidity, atonic urinary bladder, and paralytic ileus with preservation of dorsal column sensory modalities. Lesions that interrupt the innervation to the diaphragm via the C3-5 segments may lead to impairment of respiration but this is unusual. Cheshire et $a l^{6}$ reviewed 44 cases of ischaemia and infarction of the spinal cord over a 12 year period. There was a cervical level as high as C4 in only two cases, both associated with reversible mechanical cord compression (spondylosis and haematomyelia) and, whereas four patients in total had a cervical sensory level and 28 a thoracic level, no patient was described as requiring ventilatory support. The patients described in the present series therefore present a highly atypical clinical pattern. An alternative cause for spinal cord infarction is embolism of cartilaginous material from intervertebral discs. ${ }^{14-17}$ This is thought to occur due to acute vertical disc herniation leading to increased intraosseous pressure and fibrocartilage emboli from the nucleus pulposus. Many of the cases have occurred in young women and have been associated with minor trauma, sudden neck motion, or lifting. They present with pain in the neck, upper back, or occasionally in a radicular distribution. There is then a variable latent period of between 15 minutes and 48 hours until the neurological deficit of an anterior spinal artery syndrome develops to maximum severity. Once established, the neurological deficit does not usually improve significantly. The cervical cord is a common site for fibrocartilaginous embolism with 22 of the 32 reported cases occurring in this region. Involvement of high cervical cord levels and, rarely, the medulla oblongata is associated with respiratory impairment and arrest; however, long term survival with ventilatory support has 
not previously been described. In pathologically established cases radiological assessment with plain films, myelography, CT, and MRI have often been unrevealing. ${ }^{17}$ It is not clear whether patients in the present series had fibrocartilaginous emboli as a cause of their spinal cord infarction. However, the first three patients were aged 30 or younger and all presented with significant neck pain and a variable latent period of between several hours and three days before the development of severe neurological deficit and in one patient there was no respiratory improvement. It remains possible that a more conventional cause for spinal cord infarction may have occurred; certainly, patient 4 presented a different clinical and respiratory pattern more suggestive of a characteristic anterior spinal artery occlusion but, unusually, occurring at $\mathrm{C} 3 / 4$. In conclusion, infarction of the spinal cord at high cervical levels is usually due to occlusion of the anterior spinal artery and may be due to fibrocartilaginous embolism. Involvement of the descending respiratory pathways may occur. Patients may present with neck or shoulder pain but then develop a rapidly evolving tetraplegia and respiratory insufficiency culminating in respiratory arrest. Extensive lesions at C1/2 cause complete interruption of descending respiratory control leading to apnoea. Partial lesions at $\mathrm{C} 3 / 4$ cause selective interruption of automatic or voluntary pathways and give rise to characteristic respiratory patterns. The prognosis depends on the level and extent of the lesion. Complete anterior spinal artery occlusion causing infarction which extends up to $\mathrm{C} 1$ has a poor outlook whereas incomplete occlusion at C3/4 may show significant recovery of respiratory and limb function.

1 Guttman L. Spinal cord injuries, 1st ed. Oxford: Blackwell Scientific Publications 1973:182-99.

2 O'Riordan JI, Gallagher HL, Thompson AL, et al. The O'Riordan JI, Gallagher HL, Thompson AL, et al. The
clinical, cerebrospinal fluid and MRI findings in Devic's neuromyelitis optica. I Neurol Neurosurg Psychiatry 1996; 60:382-7.

3 McEvoy RD, Mykytyn I, Sajkov D, et al. Sleep apnoea in patients with quadriplegia. Thorax 1995;50:613-9.

4 Short DJ, Stradling JR, Williams SJ. Prevelance of sleep apnoea in patients over 40 years of age with spinal cord lesions. F Neurol Neurosurg Pschiatry 1992;55:1032-6.

5 Sandson TA, Friedman JH. Spinal cord infarction. Report of 8 cases and review of the literature. Medicine (Baltimore) 1989;69:282-92.

6 Cheshire WP, Santos CC, Massey EW, et al. Spinal cord infarction: etiology and outcome. Neurology 1996;47:32130 .

7 Nathan PW. The descending respiratory pathway in man. $\mathcal{F}$. Neurol Neurosurg Psychiatry 1963;26:487-99.

8 Belmusto L, Brown E, Owens G. Clinical observations on respiratory and vasomotor disturbances are related to cervical cordotomies. $\mathcal{F}$ Neurosurg 1963;20:225-32.

9 Krieger AJ, Rosomoff H. Sleep-induced apnoea. Part 1: a respiratory and autonomic dysfunction syndrome following bilateral percutaneous cervical cordotomy. $\mathcal{f}$ Neurosurg 1974;39:168-80.

10 Plum F. Neurological integration of behavioural and metabolic control of breathing. In: Parker R, ed. Breathing: Hering-Breuer centenary symposium. London: Churchill 1970:159-81.

11 Newsom Davis J. Autonomous breathing. Arch Neurol 1974; 30:480-3.

12 Howard RS, Newsom Davis J. The neural control of respiratory function. In: Crockard A, Hayward R, Hoff JT, eds. Neurosurgery - the scientific basis of clinical practice. Oxford: Blackwell, 1992:318-36.

13 Howard RS, Wiles CM, Hirsch NP, et al. Respiratory involvement in multiple sclerosis. Brain 1992;115:479-94.

14 Srigley JR, Lambert CD, Bilbao JM, et al. Spinal cord infarction secondary to intervertebral disc embolism. Ann Neurol 1981;9:296-300.

15 Banerjee AK, Deodhar SD. Cartilage embolism of the spinal cord. F Neurol Neurosurg Psychiatry 1989;52:1201-2.

16 Case records of The Massachusetts General Hospital. Case 5-1991. N Engl f Med 1991;324:322-32.

17 Tosi L, Rigoli G, Beltramello A. Fibrocartilaginous embolism of the spinal cord: a clinical and pathogenetic embolism of the spinal cord: a clinical and pathogenetic
reconsideration. F Neurol Neurosurg Psychiatry 1996;60:5560. 